\title{
Laser phase errors in seeded free electron lasers
}

\author{
D. Ratner, A. Fry, G. Stupakov, and W. White \\ SLAC, Menlo Park, California 94025, USA \\ (Received 7 December 2011; published 12 March 2012)
}

\begin{abstract}
Harmonic seeding of free electron lasers has attracted significant attention as a method for producing transform-limited pulses in the soft $\mathrm{x}$-ray region. Harmonic multiplication schemes extend seeding to shorter wavelengths, but also amplify the spectral phase errors of the initial seed laser, and may degrade the pulse quality and impede production of transform-limited pulses. In this paper we consider the effect of seed laser phase errors in high gain harmonic generation and echo-enabled harmonic generation. We use simulations to confirm analytical results for the case of linearly chirped seed lasers, and extend the results for arbitrary seed laser envelope and phase.
\end{abstract}

DOI: $10.1103 /$ PhysRevSTAB.15.030702

PACS numbers: 41.60.Cr, 42.60.Jf, 42.62.-b, 42.55.Vc

\section{INTRODUCTION}

The recent success of self-amplified spontaneous emission (SASE) free electron lasers (FELs) has led to x-ray sources of unprecedented brightness [1,2]. However, some applications still require higher power (e.g., [3,4]), and the poor longitudinal coherence of SASE FELs can inhibit $\mathrm{x}$-ray optimization and degrade experimental results. To improve control over the spectral and temporal x-ray properties, there is strong interest in seeding FELs at high harmonics of optical or UV lasers. Beam line users are particularly interested in the minimal bandwidth and simple temporal structure of transform-limited $\mathrm{x}$-ray pulses. In this paper, we consider how errors in the seeding process may affect production of transform-limited x-ray pulses.

There are numerous challenges for seeding schemes, and previous theoretical and experimental studies have focused on a wide variety of accelerator and FEL requirements. In particular, it is well known that harmonic seeding schemes must contend with increasingly strict electron beam tolerances as the harmonic number increases. Initial errors that are insignificant compared to the seed wavelength may be large relative to a much shorter wavelength harmonic. For example, harmonic multiplication amplifies electron shot noise, which can overwhelm the external seeding source [5-8]. More recently, attention has turned to errors from the seed laser itself (see, e.g., [9]). Without sufficient control of the initial seed laser phase, the x-ray pulse acquires longitudinal structure; if sufficiently far from the transform limit, seeding may have little or no benefit compared to SASE FELs.

In this paper, we study the effects of laser phase errors on the seeded electron density. Recent papers have used analytical methods to derive the sensitivity of seeding schemes

Published by the American Physical Society under the terms of the Creative Commons Attribution 3.0 License. Further distribution of this work must maintain attribution to the author(s) and the published article's title, journal citation, and DOI. to quadratic laser phase $[10,11]$. Here we develop simulations to observe the increase in phase errors as a function of harmonic number in high gain harmonic generation (HGHG) and echo-enabled harmonic generation (EEHG). We show that simulations match analytical results for the case of quadratic phase, and extend the simulations to arbitrary higher order spectral phase. Finally, we consider a practical HGHG case to estimate the required level of noise control in future seeded FELs.

\section{SCHEMATIC DESCRIPTION OF HARMONIC PHASE MULTIPLICATION}

As a simple example of seeding, we begin with the case of HGHG driven by a temporally flattop laser pulse. In idealized HGHG [12], the laser produces an energy modulation in the electron beam, and a dispersive region converts the energy modulation into a density modulation. After the combined modulation and dispersion, the electron beam has periodic density spikes separated by the laser wavelength, $\lambda_{L}$; i.e., the electrons are "bunched" at a wave vector, $k_{1}=k_{L}$, with laser wave vector $k_{L} \equiv$ $2 \pi / \lambda_{L}$. If the density spikes are short compared to the harmonic wavelength, $\lambda_{L} / H$, the electrons are also bunched at the harmonic wave vector $k_{H}=H k_{1}$, for a given harmonic, $H$. [Later we will quantify the degree of density modulation from the bunching factor, Eq. (5).]

In a realistic laser pulse, the wavelength varies as a function of time. As the wavelength changes, the resulting separation of electron density spikes also shifts from the central wavelength, as illustrated in Fig. 1. If the wavelength varies by $\Delta \lambda$ across the bunch, then the peak electron bunching will vary by $\Delta k=2 \pi \Delta \lambda / \lambda^{2}$. The relative variation in wave vector is constant at all harmonics, so $\Delta k_{H} / k_{H}=\Delta k_{1} / k_{1}$.

The electron bunching determines the FEL radiation, so an increase in $\Delta k_{H}$ increases the radiated bandwidth as well. If the seed laser pulse is far from the transform limit, then we expect the total radiated bandwidth to grow as 


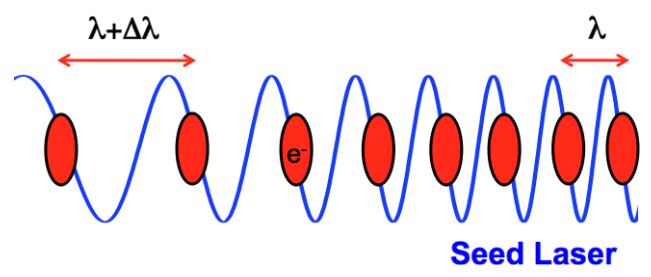

FIG. 1. Cartoon illustrating the effect of seed phase errors on HGHG electron bunching. A time-varying wavelength in the seed laser (blue line) results in a varying separation of the bunched electrons (red bunches).

$$
\Delta k_{H} \approx H \Delta k_{1} .
$$

To quantify the effect of wavelength variation in the seed laser, we calculate the time-bandwidth product (TBP) of the FEL,

$$
\mathrm{TBP}=c \Delta T_{\mathrm{FEL}} \Delta k_{\mathrm{FEL}},
$$

from the pulse duration $\Delta T_{\mathrm{FEL}}$, bandwidth $\Delta k_{\mathrm{FEL}}$, and speed of light $c$. For a given spectral distribution, the minimal TBP corresponds to a transform-limited pulse. As the TBP grows, the seeded FEL characteristics revert to those of a SASE pulse.

For a flattop seed laser with a small linear variation in wavelength, all harmonics have the same pulse length. From Eq. (1) we then expect the TBP to grow linearly with harmonic number. [This holds only for small changes in wavelength, when the entire laser pulse satisfies the HGHG bunching condition, Eq. (10).]

While it is possible to produce seed laser pulses with nearly minimal TBP, at sufficiently large harmonic numbers the growth in $\Delta k_{\mathrm{FEL}}$ will lead to pulses far from the transform limit. For realistic laser envelope and phase, the harmonic amplification is more complicated, but the TBP still grows with increasing harmonic number. The following sections develop these more realistic cases in detail.

\section{HGHG WITH SPECTRAL PHASE ERRORS}

\section{A. Laser in spectral domain}

Experimental laser measurements are predominantly spectral, so it is convenient to describe the laser pulse using the electric field in the spectral domain,

$$
\tilde{\mathcal{E}}(k)=\tilde{E}(k) e^{-i \phi(k)},
$$

with spectral intensity $\tilde{E}(k)$ and phase

$$
\phi(k)=\sum_{n=2}^{\infty} \frac{\phi_{n}}{n !}\left(k-k_{0}\right)^{n} .
$$

(We ignore the $\phi_{0}$ and $\phi_{1}$ terms, which represent the carrier-envelope offset and the envelope temporal delay respectively, and are not relevant to this analysis.) A transform-limited pulse by definition has minimal TBP and flat spectral phase, $\phi(k)=0$. Realistic laser pulses will have non-negligible spectral phase, and these phase terms produce longer pulses with greater intensity fluctuation in the time domain; i.e., pulses farther from the transform limit.

We start by considering laser pulses with Gaussian spectrum, $\tilde{E}(k)$, and only quadratic spectral phase, $\phi_{2}$, as these are easily studied analytically in the time domain. With simulations, we then extend the results to include arbitrary spectral envelope and phase.

Throughout the paper, we will assume the laser pulse is short compared to the electron bunch. Production of sufficient laser power at short wavelengths is currently a challenging limitation for seeding x-ray FELs and the long electron bunch ensures there is no wasted laser pulse energy. However, there are advantages to a long laser pulse. Phase control is simpler for a narrow bandwidth laser, and a short electron bunch will sample the phase only at the center of a long seed laser (Sec. III E). The complementary case of a short electron bunch and long laser pulse was described recently in Ref. [11].

\section{B. Electron bunching factor}

The final electron distribution is responsible for the properties of the FEL. To estimate the FEL radiation at wave vector $k$, we define the averaged electron bunching factor:

$$
b(k) \equiv \frac{1}{N_{T}} \sum_{j=1}^{N_{T}} e^{-i k \bar{z}_{j}},
$$

where the sum is over the final longitudinal position, $\bar{z}$, of all $N_{T}$ electrons in the bunch. We can also define a local bunching factor by summing over a single wavelength slice of the beam. In this case, we change the normalization of Eq. (5) to the number of electrons in the local slice, $N_{\text {slice }}(z)$,

$$
b_{\text {slice }, k}(z) \equiv \frac{1}{N_{\text {slice }}(z)} \sum_{j=1}^{N_{\text {slice }}(z)} e^{i k \bar{z}_{j}}
$$

In HGHG and EEHG, the seeded bunching factor largely determines the FEL characteristics at saturation. For example, the length of the slice bunching $b_{\text {slice, } k}(z)$ determines the duration of the FEL pulse $\Delta T_{\mathrm{FEL}}$. The width of a harmonic in the averaged bunching $b(k)$ determines the FEL bandwidth $\Delta k_{\mathrm{FEL}}$. From the product of the rms FEL duration $\Delta T_{\mathrm{FEL}}$ and bandwidth $\Delta k_{\mathrm{FEL}}$, we find the TBP of the FEL.

We can also define a spectral phase of the electron bunch from the argument of the averaged bunching factor,

$$
\phi_{\mathrm{e}^{-}}(k)=\operatorname{Arg}[b(k)] .
$$

The electron spectral phase is directly analogous to the laser spectral phase [Eq. (4)].

Central to all calculations is the assumption that the seeded bunching factor determines the properties of the 
FEL radiation. The FEL can affect the electron spectral phase in two ways. First, the FEL process is itself a narrow band amplifier, which can in principle change the output radiation properties. However, for Linac Coherent Light Source the FEL bandwidth is as large as $\Delta k_{\mathrm{SASE}} / k=$ $0.1 \%$. By contrast, seeded FELs hope to generate bandwidths as small as $\Delta k_{\mathrm{SEED}} / k=0.01 \%$, so we assume that $\Delta k_{\text {SEED }} \ll \Delta k_{\text {SASE }}$; in the opposite limit, there is little purpose to seeding as the SASE process will dominate. For the case of short-pulse seeding when $\Delta k_{\text {SEED }} \lesssim$ $\Delta k_{\mathrm{SASE}}$, the FEL process could limit the bandwidth broadening of large phase errors.

Second, dispersion in the undulators can compress the electron beam, altering $b(k)$ (see, e.g., [13]). Variation in the relative energy of the electron beam, $h(z) \equiv d p / d z$, leads to a changing seeded wavelength $\lambda \rightarrow \alpha(z) \lambda$, with compression factor

$$
\alpha(z) \approx 1+R_{\text {und }}^{(56)} h(z),
$$

and dispersion $R_{\text {und }}^{(56)}=2 N_{u} \lambda$ over $N_{u}$ undulator periods. While a constant $\alpha(z)$ shifts $\lambda$, if $\alpha(z)$ varies across the bunch, the final $\mathrm{x}$-ray pulse may acquire additional temporal structure. With a sufficiently flat-phase space, $\left|R_{\text {und }}^{(56)} h(z)\right|<\Delta k_{\mathrm{SEED}} / k$, we can ignore all compression effects.

\section{Second order spectral phase, analytical approach}

We are now ready to consider the effects of laser spectral phase on the electron bunching. A seed laser pulse with a Gaussian spectral amplitude of rms width, $\sigma_{k}$, and second order spectral phase, $\phi_{2}$, transforms into itself in the time domain; i.e., a pulse with a Gaussian $E$ field

$$
\mathcal{E}(z)=E_{0} e^{-z^{2} / 2 \sigma_{L}^{2}} e^{i\left(k_{L} z+\alpha_{2} z^{2}\right)},
$$

where the temporal envelope $\sigma_{L}$ and second order temporal phase $\alpha_{2}$ are determined by the spectral equivalents, $\sigma_{k}$ and $\phi_{2}$. The derivative of the phase determines the instantaneous wavelength, so quadratic temporal phase $\alpha_{2}$ produces a linear change in wavelength. Because the time-domain pulse has a simple analytical form, we can solve for the resulting electron bunching factor analytically as well. (The derivation for the following section can be found in Ref. [10].)

To calculate the averaged bunching factor $b(k)$, we assume a longitudinally uniform distribution with an energy spread of $\sigma_{p}$. In the case of HGHG, the final particle position is given by $\bar{z}=z+R_{56}\left[p+A_{0} \cos \left(k_{L} z\right)\right]$, with initial longitudinal position and energy, $z, p$, dispersion $R_{56}$, and amplitude of laser-induced energy modulation $A_{0}$. To produce bunching at harmonic $H \gg 1$, we require the standard HGHG condition $A_{0} \gtrsim H \sigma_{p}$, and optimize bunching at the laser center with

$$
R_{56} \approx\left(1+0.81 H^{-2 / 3}\right) / A_{0} k_{L}
$$

To find the bunching factor at wave vector $k_{H}+\delta k_{H}$ near a harmonic $k_{H}$, we then integrate Eq. (5) over the electron distribution to find the averaged bunching factor [10]:

$$
\begin{aligned}
b_{H}(\delta k) \propto & \sigma_{L} e^{-H^{2} r^{2}\left(1+0.81 H^{-2 / 3}\right)^{2} / 2 A_{0}^{2}} \\
& \times G_{H}^{(\mathrm{HGHG})}\left(\delta k \sigma_{L} / H, H \beta \sigma_{L}^{2} / \lambda^{2}, r\right),
\end{aligned}
$$

with

$$
G_{H}^{(\mathrm{HGHG})}(x, y, r) \equiv \int_{-\infty}^{\infty} d \xi e^{i x \xi+i y \xi^{2}} J_{H}\left[r\left(H+0.81 H^{-1 / 3}\right) e^{-\xi^{2} / 2}\right],
$$

where $\beta \equiv \alpha_{2} / 2 k_{L}^{2}$ is the dimensionless second order phase and $r \sim 1$ optimizes the bunching factor near the peak of the laser pulse. From $b_{H}(\delta k)$ we can determine both the bandwidth $\Delta k_{\mathrm{FEL}}$ and the spectral phase $\phi_{\mathrm{e}^{-}}(k)$, as a function of harmonic number.

\section{Second order spectral phase, simulation}

To extend the study to higher order spectral phase, we developed a 1D particle simulation of an HGHG seeding scheme. Starting from a longitudinally uniform electron beam (much longer than the seed laser pulse) with a Gaussian energy distribution, we apply an energy modulation from the electric field of a laser pulse [e.g., Eq. (9)]. We then follow the modulation with a dispersive region, and finally calculate the bunching factor [Eq. (5)] at the exit of the HGHG seeding.

Using the metrics described in Sec. III B, we calculate the rms TBP as a function of harmonic number. Figures 2 and 3 show the bunching factor and TBP for a laser pulse with quadratic spectral phase. We confirm that laser phase errors widen the bunching bandwidth at high harmonics, as predicted by Eq. (12).

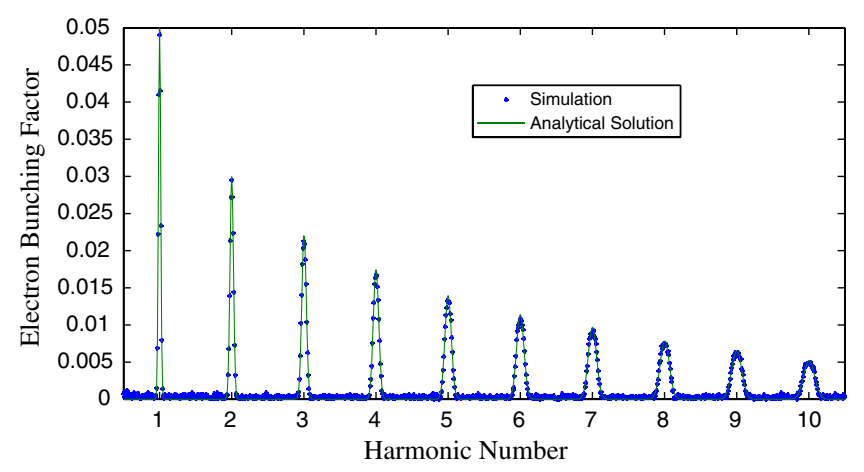

FIG. 2. Electron bunching factor for a Gaussian seed laser pulse with quadratic phase. The seed laser pulse has phase $\phi\left(\sigma_{k}\right)=\pi$, amplitude $A_{0}=30 \sigma_{p}$, and rms bandwidth $\sigma_{k} / k_{L}=10 \%$. Amplitude of the bunching factor is small due to averaging over a long electron bunch of uniform length $L=$ $10 \sigma_{L}$ [Eq. (5)]. Simulated bandwidths (blue) reproduce the analytical result [Eq. (12) in green, scaled to match the bunching amplitude]. As expected, the peaks broaden at higher harmonics. 


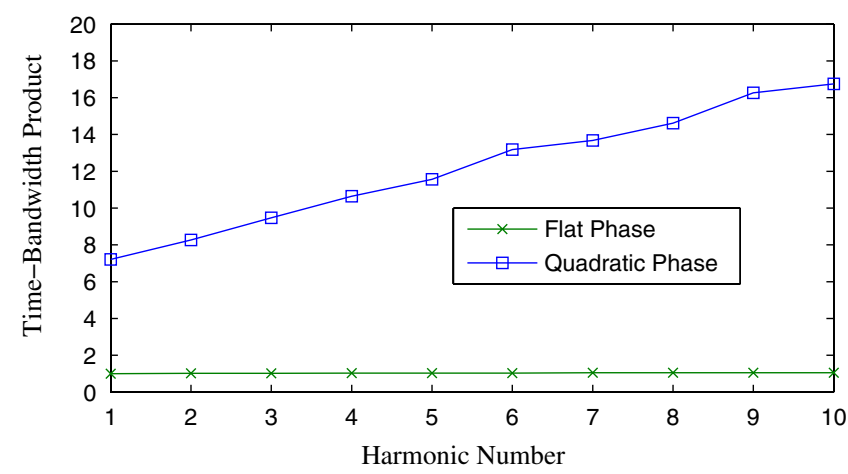

FIG. 3. The rms TBP, $c \Delta T_{\mathrm{FEL}} \Delta k_{\mathrm{FEL}}$, for the electron bunching factor of Fig. 2. The TBP increases at higher harmonics (blue squares), but slower than would be expected from a flattop pulse. For comparison, a transform-limited pulse with flat phase has minimal TBP at all harmonics (green crosses).

The normalization of the average bunching factor, Eq. (5), depends on the total number of electrons in the entire bunch, while the shorter seed laser determines the length of the final radiated pulse. As a result, the average bunching factor may be small even though the slice bunching factor, Eq. (6), is large where the seed laser overlaps the electrons. Here we are interested primarily in the width and phase of each bunching peak, rather than the overall amplitude.

Figure 4 shows the spectral phase $\phi_{\mathrm{e}^{-}}(k)$ for the first ten harmonics, calculated from both simulations and Eq. (11). When the seed pulse has quadratic phase, the electron bunching factor also shows quadratic phase. As expected, the phase increases as a function of harmonic number.

In principle, it is possible to correct for a linear change in wavelength (or "chirp") by compressing the radiated x-ray pulse with a diffraction grating. Indeed, chirp-pulse amplification (CPA) schemes have long been proposed for generating short, high power FEL pulses (see, e.g., [14-17]). In the case of a CPA FEL, the TBP of the seeding overestimates the TBP of the final compressed $\mathrm{x}$-ray pulse.

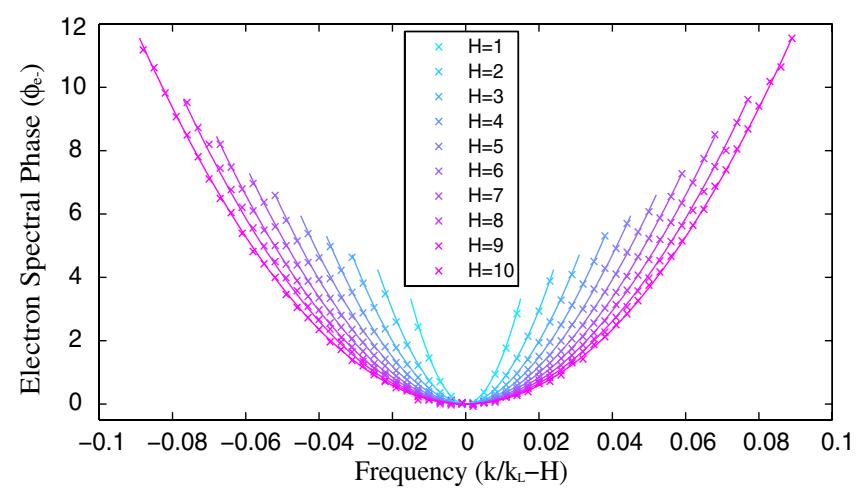

FIG. 4. Spectral phase of the electron bunching factor from Fig. 2 for the first ten harmonics. Solid line calculated from Eq. (12), with crosses taken from simulations. At higher harmonics the curves are wider due to the increasing bandwidth.
At present, grating efficiencies near $1 \mathrm{~nm}$ are too low for CPA to increase the peak power, but advances in blazed gratings may make CPA schemes more effective in the future (see, e.g., [18]).

\section{E. Pulse shortening}

The increase in TBP for Gaussian pulses is not as large as predicted by the flattop argument in Sec. II. The flattop and Gaussian cases differ primarily due to the effect of harmonic pulse shortening. High harmonic bunching relies on the creation of sharp density spikes; when the laser $E$-field amplitude drops away from the peak of a Gaussian pulse, the HGHG bunching condition [Eq. (10)] is suboptimal and broadens the density spikes. The widening density spikes cut off higher harmonics first, leading to increasingly short pulses as the harmonic number increases. Figure 5 shows the local slice bunching factor [Eq. (6)] as a function of position along the bunch; as expected, the pulse lengths are shorter at higher harmonics. In Fig. 6 we confirm that in HGHG from a Gaussian seed laser, the pulse length is approximately proportional to $H^{-1 / 3}$ [10]. By contrast, when seeding from a flattop pulse, the width of density spikes is independent of longitudinal position, and all harmonics have the same duration.

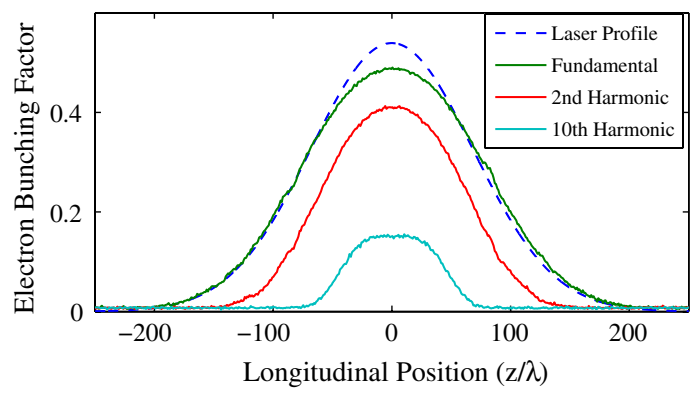

FIG. 5. Slice electron bunching factor and laser $E$ field as a function of longitudinal position. Each bunching factor point corresponds to a slice of the electron bunch of width $\lambda_{L}$ [Eq. (6)].

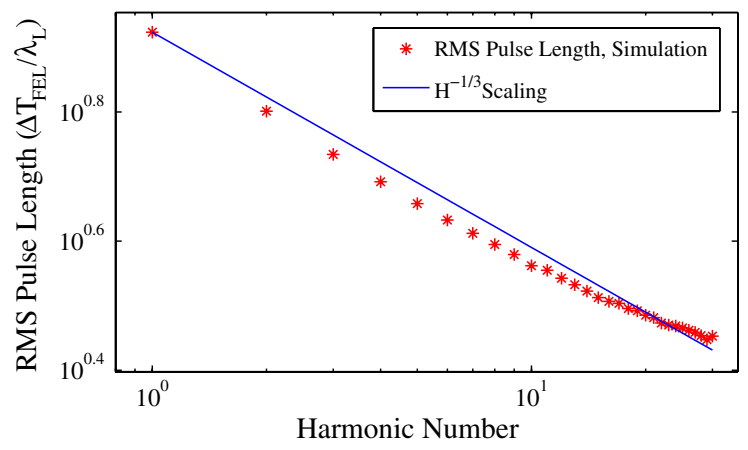

FIG. 6. Pulse length as a function of harmonic number. Simulation pulse lengths determined from the electron bunching factor (stars) follow the expected $H^{-1 / 3}$ scaling (line). 
Pulse shortening changes the harmonic spectral phase; while the fundamental bunching follows the seed laser amplitude and phase, the harmonic bunching samples phase only from the center of the seed pulse. As a result, the harmonic spectral phase from a Gaussian seed laser is smaller than that from an equivalent flattop pulse. Whereas Sec. II predicts an increase in phase proportional to harmonic number [Eq. (1)], Figs. 3 and 4 show that a Gaussian envelope produces weaker growth in bandwidth and TBP [Eq. (12)].

While pulse shortening has the beneficial effect of limiting the harmonic phase errors, the shorter pulses result in a larger coherent bandwidth and lower spectral brightness. To produce a narrow bandwidth FEL requires long seed lasers with high pulse energy. Though the phase of the long pulse may be easier to control, it is not possible to use arbitrarily long laser pulses as the corresponding increase in pulse energy will push already challenging parameters.

\section{F. Arbitrary spectral phase, simulation}

A realistic laser pulse contains non-negligible spectral phase beyond the 2nd order. Because of the difficulty of writing an analytical time-domain expression for arbitrary spectral phase, we use simulations to study harmonic amplification of such pulses.

Figure 7 shows the 10th harmonic electron spectral phase, $\phi_{\mathrm{e}^{-}}(k)$, from seed lasers with 2 nd through 5 th order spectral phase. We note that the odd order phases have less impact on the electron bunch than the even orders. Figure 8 illustrates the reasoning as follows: odd order spectral phase produces side pulses in the time domain, with a $\pi$ flip in the temporal phase between each pulse. In Sec. III E we found that at high harmonics, $b_{\text {slice, }}(z)$ is significant only in the center of the laser pulse, where the temporal

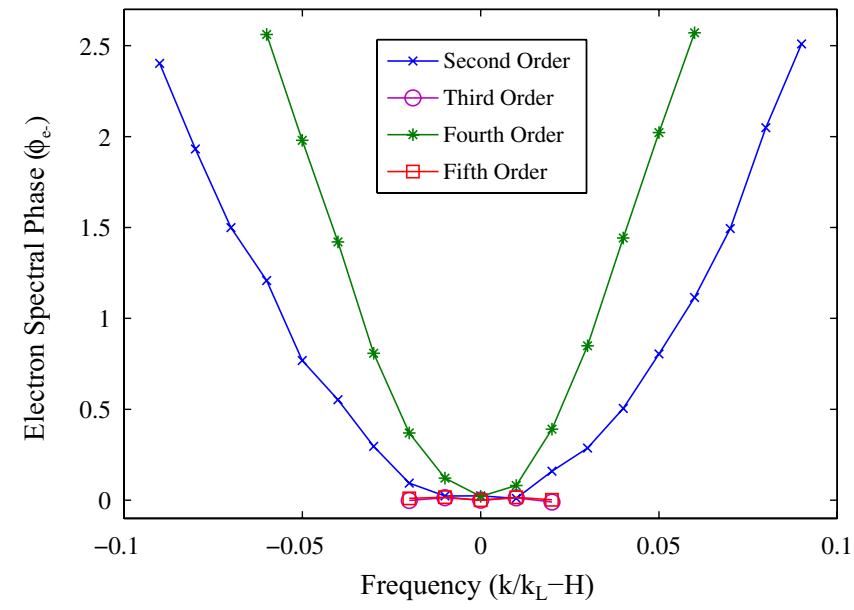

FIG. 7. Electron spectral phase, $\phi_{\mathrm{e}^{-}}$, at the 10th harmonic for seed laser pulses with 2 nd through 5 th order spectral phase. For each curve the spectral phase of the laser seed has $\phi\left(\sigma_{k}\right)=$ $\phi_{n} \sigma_{k}^{n} / n !=1$ [Eq. (4)]. The bandwidth $\Delta k_{\mathrm{FEL}}$ determines the plotted range; the odd order cases produce narrower bandwidths.

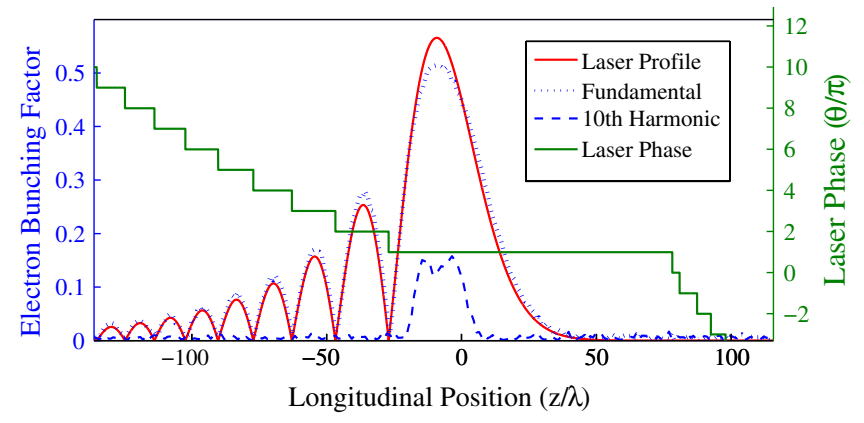

FIG. 8. Slice bunching factor [Eq. (6)] as a function of position for the fundamental and 10th harmonic. Third order spectral phase on the seed laser produces side pulses on the time-domain $E$ field (solid red line). The temporal phase (solid green line) is flat within each pulse, but jumps by $\pi$ between pulses. The 10th harmonic bunching factor (dashed blue line) exists only in the center of the largest pulse, and consequently does not pick up the third order phase.

phase is constant. This central region dominates the averaged bunching $b(k)$, so $\phi_{3}$ and $\phi_{5}$ make little contribution to $\phi_{\mathrm{e}^{-}}(k)$.

We find that all even orders contribute at approximately the same level when $\phi_{n} \sigma_{k}^{n} / n !=1$. Controlling spectral phase becomes increasingly difficult at higher orders. However, for long pulses with narrow bandwidths $\sigma_{k}$ (e.g., durations above $\sim 50 \mathrm{fs}$ ), we expect $\phi_{n} \sigma_{k}^{n} / n ! \ll 1$ for $n>5$ and can ignore the higher orders. For shorter pulses (e.g., durations below $20 \mathrm{fs}$ ), it may be necessary to control the phase for $n=6$ as well.

The loose constraints on the odd order phase may aid in production of transform-limited pulses. For example, canceling only the even order seed laser phase will reduce the complexity of the optical setup. Alternatively, it may be beneficial to treat the laser phase as a total minimization problem; rather than separately minimizing each order, it is possible to collectively select all orders to minimize the TBP of the FEL. This collective approach is analogous to methods used in the production of transform-limited laser pulses $[19,20]$.

\section{EEHG WITH SPECTRAL PHASE ERRORS}

The EEHG seeding mechanism [21] shares many similarities with HGHG. In the standard EEHG arrangement, the first laser-chicane combination filaments the electron beam in phase space. The second laser-chicane stage then simultaneously bunches each filament, resulting in multiple density spikes within each seed wavelength.

The spectral phase on the seed laser will affect the two stages differently. On the first laser pulse, spectral phase distorts the separation of the filaments, so that the density spikes do not fall exactly at the harmonic spacing (Fig. 9). The distortion reduces the bunching factor, but because the second laser still phase locks each set of density spikes, there is relatively little effect on the TBP. (If the reduced 


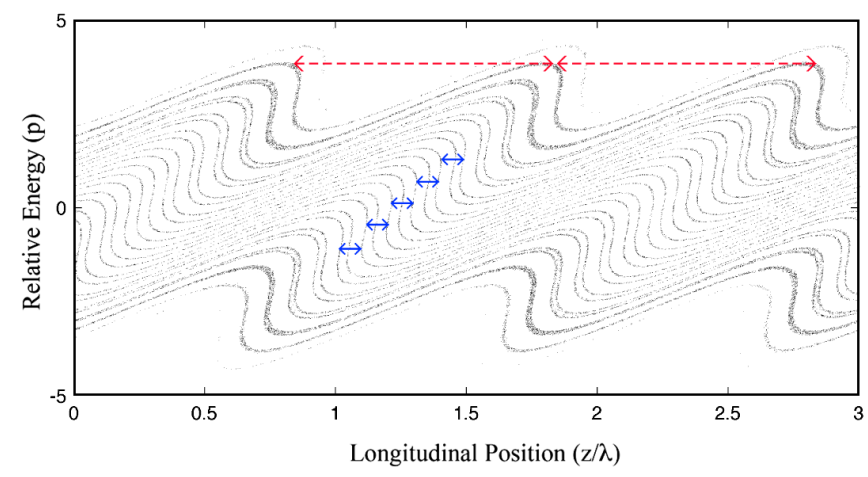

FIG. 9. Schematic of EEHG phase space. Phase errors on the first stage distort the separation of density spikes within a single modulation wavelength (solid blue arrows), but the length of each group (dotted red arrows) is phase locked by the second stage. Phase errors on the second stage, by contrast, can change the final seeded wavelength.

bunching factor also shortens the pulse length, there may be coherent broadening of the bandwidth.)

The second stage of EEHG is similar to the HGHG process, but with the energy separation of the filaments determining the final harmonic number. Following the approach of Sec. III, we again find that the electron bunching factor follows the spectral phase of the seed laser. If we assume a flattop laser pulse in the first stage and a Gaussian pulse of length $\sigma_{L 2}$ in the second stage, the increase in electron bunching factor bandwidth is given by $G_{H}^{(\mathrm{EEHG})}\left[\delta k \sigma_{L 2} / H,(H+1) \beta \sigma_{L 2}^{2} / \lambda^{2}\right][10]$, with

$$
\begin{aligned}
G_{H}^{(\mathrm{EEHG})}(x, y) \equiv & \int_{-\infty}^{\infty} d \xi e^{i x \xi+i y \xi^{2}} J_{H+1}\{r[(H+1) \\
& \left.\left.+0.81(H+1)^{-1 / 3}\right] e^{-\xi^{2} / 2}\right\},
\end{aligned}
$$

analogous to Eq. (12) for the case of HGHG.

As for HGHG, we use the simulations to expand on the analytical results. In Fig. 10 we simulate an EEHG scheme at the 10th harmonic, using pulses with Gaussian envelope and arbitrary spectral phase for both laser stages. We consider three cases: flat phase on both stages, quadratic spectral phase on only the first stage, and quadratic spectral phase on only the second stage. As expected, a chirp on the second stage increases the electron bunching factor bandwidth, while a chirp on the first stage only decreases the overall bunching factor. We note that the increase in TBP is actually worse than suggested by Fig. 10; the spectral phase on the second stage stretches the laser pulse in time, so the increase in bandwidth is accompanied by an additional increase in pulse length.

It is possible to treat the arbitrary case of Fig. 10 by extending the approach of Ref. [10]. However, we note that the approximate solution given in Eq. (13) predicts the simulated increase in bandwidth reasonably well (Fig. 10), validating the assumption of a flattop pulse with flat phase in the first stage.

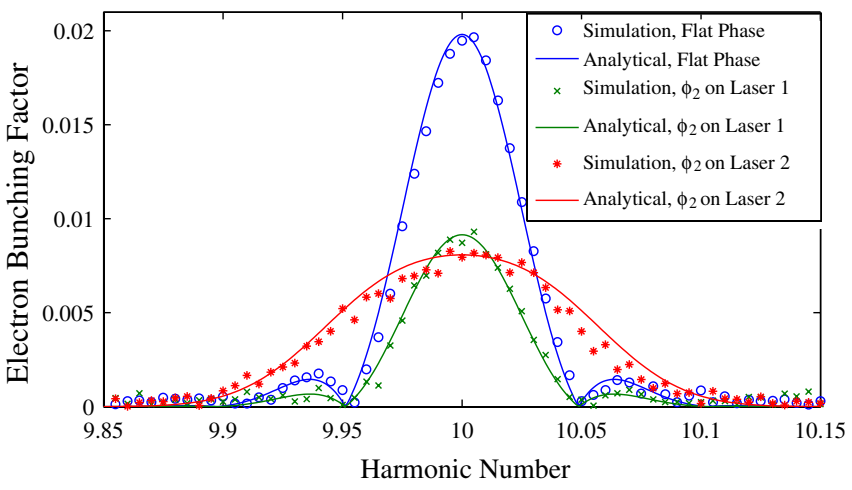

FIG. 10. Averaged bunching factor for 10th harmonic EEHG with Gaussian envelope for both laser stages. Quadratic phase on the first seed laser $\left(\phi_{2} \sigma_{k}^{2} / 2=1\right)$ reduces the bunching factor, but does not broaden the bandwidth. The same quadratic phase on the second seed laser increases the bandwidth and TBP as found for HGHG. Solid lines show the numerical integral, Eq. (13).

\section{PRACTICAL EXAMPLE}

We conclude by simulating a practical example using an $800 \mathrm{~nm}$ laser pulse. Table I gives experimentally measured spectral parameters from an ultrafast Ti:sapphire amplifier (the Coherent Legend Elite USX). The pulse length of $22 \mathrm{fs}$ is close to the transform-limited (flat phase) pulse length of $20 \mathrm{fs}$. Despite the nearly transform-limited initial seed laser pulse, Fig. 11 shows that the electron bunching factor at the 30th harmonic is approximately 3 times the transform limit.

We can use the parameters of Table I to give a rough estimate of the phase control required for HGHG and EEHG seeding of transform-limited pulses. Figure 12 shows the slice bunching factor (30th harmonic) vs time for the cases of flat phase, measured phase, and double measured phase. If the phase errors increase beyond the level of Table I by just a factor of 2 , the formerly transformlimited pulse starts to acquire temporal modulations. As a result, we expect that it will be necessary to include phase control at the level of Table I to preserve the temporal characteristics of the seed laser. While this example

TABLE I. Measured parameters for a nearly transform-limited $800 \mathrm{~nm}$ pulse. The fourth order phase dominates the FEL performance.

Measured laser pulse

Central wavelength

$800 \mathrm{~nm}$

Bandwidth (fwhm)

$73 \mathrm{~nm}$

Pulse duration

Second order phase (GDD)

$22 \mathrm{fs}$

Third order phase (TOD)

Fourth order phase (FOD)

$0.5 \mathrm{fs}^{2}$

Fifth order phase (5OD)
$2.4 \times 10^{3} \mathrm{fs}^{3}$

$-4.6 \times 10^{4} \mathrm{fs}^{4}$

$-1.2 \times 10^{6} \mathrm{fs}^{5}$ 


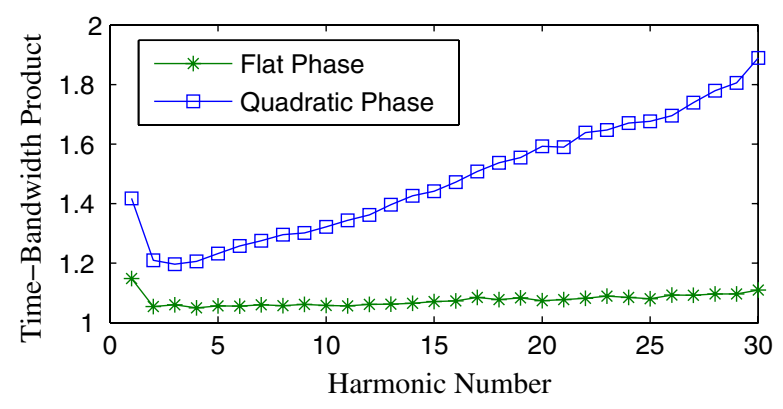

FIG. 11. The rms TBP vs harmonic number for the pulse parameters given in Table I. Even for this nearly transformlimited seed pulse, the TBP at the 30th harmonic (blue squares) is almost 3 times the transform-limited flat-phase case (green stars).

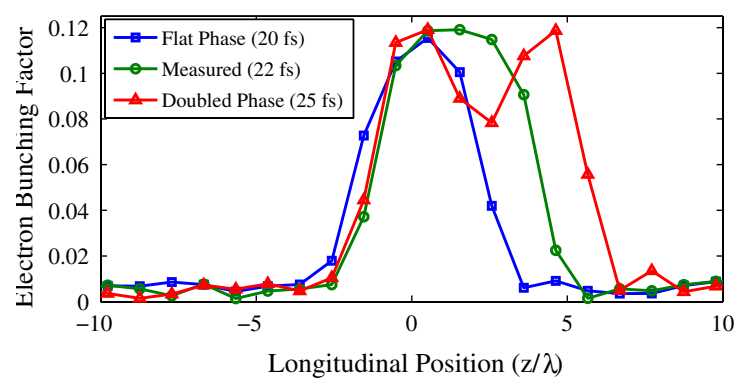

FIG. 12. Slice electron bunching vs time for parameters of Table I. Bunching is shown for the 30th harmonic of laser pulses simulated with flat phase (blue squares), measured phase (green circles), and twice the measured phase (red triangles).

assumes an $800 \mathrm{~nm}$ laser, the results scale to the shorter seed wavelengths of interest to $\mathrm{x}$-ray seeding.

We conclude that it will be difficult to achieve transform-limited pulses beyond the 50th harmonic, and seeding of $\mathrm{x}$-ray pulses at $1 \mathrm{~nm}$ will require laser phase control at wavelengths below $50 \mathrm{~nm}$. It is currently possible to measure pulse characteristics into the XUV range (see, e.g., [22]), but there is still a need to develop short wavelength phase control to the level currently achievable at $800 \mathrm{~nm}$.

Alternatively, a single harmonic of a high harmonic generation (HHG) source could be used as a seed. Single harmonics of HHG sources have been measured with flat spectral phase at wavelengths below $100 \mathrm{~nm}[23,24]$. However, we note that the power level required by multiplicative seeding schemes such as HGHG and EEHG pushes the current state of the art for a single HHG harmonic.

\section{CONCLUSION}

We have studied the effect of the seed laser phase on HGHG and EEHG schemes. Using analytical results and simulations, we find that the electron bunching factor copies the seed laser spectral phase. The electron spectral phase increases with harmonic number, but pulse narrowing due to the laser envelope decreases the phase growth, especially for odd order spectral phase. The pulse narrowing may aid in the production of transform-limited pulses, but will also increase laser energy requirements. We simulate a case with realistic laser parameters and find a seeded electron beam at the 30th harmonic at approximately 3 times the transform limit. We conclude that seeding near transform-limited pulses in the soft $\mathrm{x}$-ray regime will require development of new methods for phase measurement and control of short wavelength lasers or HHG sources. The required level of phase control is on par with that currently available at $800 \mathrm{~nm}$.

\section{ACKNOWLEDGMENTS}

We thank Y. Feng, Z. Huang, and P. Hering for helpful discussions. This work is supported by Department of Energy Contract No. DE-AC02-76SF00515.

[1] P. Emma et al., Nature Photon. 4, 641 (2010).

[2] H. Tanaka, in Proceedings of IPAC2011 (San Sebastian, Spain, 2011).

[3] H. Chapman et al., Nature (London) 470, 73 (2011).

[4] M. Seibert et al., Nature (London) 470, 78 (2011).

[5] E. L. Saldin, E. A. Schneidmiller, and M. V. Yurkov, Opt. Commun. 202, 169 (2002).

[6] Z. Huang, in Proceedings of the 2006 FEL Conference (Berlin, Germany, 2006), p. 130.

[7] G. Stupakov, in Proceedings of the 2010 FEL Conference (Malmo, Sweden, 2010).

[8] G. Stupakov, Z. Huang, and D. Ratner, in Proceedings of the 2010 FEL Conference (Malmo, Sweden, 2010).

[9] K. Hacker and H. Schlarb, DESY Report No. TESLA-FEL 2011-05, 2011.

[10] G. Stupakov, SLAC Report No. SLAC-PUB-14639, 2011.

[11] G. Geloni, V. Kocharyan, and E. Saldin, arXiv:1111.1615v1.

[12] L. H. Yu, Phys. Rev. A 44, 5178 (1991).

[13] Y. Ding, Z. Huang, and R. Ruth, Phys. Rev. ST Accel. Beams 13, 060703 (2010).

[14] G. T. Moore, Phys. Rev. Lett. 60, 1825 (1988).

[15] L. H. Yu, E. Johnson, D. Li, and D. Umstadter, Phys. Rev. E 49, 4480 (1994).

[16] C. Pellegrini, Nucl. Instrum. Methods Phys. Res., Sect. A 445, 124 (2000).

[17] A. Doyuran et al., Phys. Rev. ST Accel. Beams 7, 050701 (2004).

[18] R. K. Heilmann, M. Ahn, A. Bruccoleri, C. Chang, E. M. Gullikson, P. Mukherjee, and M. Schattenburg, Appl. Opt. 50, 1364 (2011).

[19] S. Kane and J. Squier, J. Opt. Soc. Am. B 14, 1237 (1997). 
[20] J. Squier, C. P. J. Barty, F. Salin, C. Le Blanc, and S. Kane, Appl. Opt. 37, 1638 (1998).

[21] G. Stupakov, Phys. Rev. Lett. 102, 074801 (2009).

[22] F. Quéré, J. Itatani, G. L. Yudin, and P. B. Corkum. Phys. Rev. Lett. 90, 073902 (2003).
[23] Y. Mairesse, O. Gobert, P. Breger, H. Merdji, P. Meynadier, P. Monchicourt, M. Perdrix, P. Salières, and B. Carré, Phys. Rev. Lett. 94, 173903 (2005).

[24] A. Kosuge, T. Sekikawa, X. Zhou, T. Kanai, S. Adachi, and S. Watanabe, Phys. Rev. Lett. 97, 263901 (2006). 